\title{
LA PERVIVENCIA DEL MITO ARTÚRICO EN EL CINE Y EN LOS VIDEOJUEGOS
}

\author{
Alberto ASLA \\ Universidad Nacional de Mar del Plata \\ Verónica BARRAGÁN
}

Universidad Nacional del Centro de la Provincia de Buenos Aires

\section{RESUMEN}

El presente artículo se propone, en primer lugar, analizar la figura del rey Arturo y los Caballeros de la Mesa Redonda, trabajando sobre los principales ejes que conforman las tradicionales historias mitológicas. En una segunda instancia, se abordará el imaginario artúrico en las películas Excalibur y King Arthur: Legend of theSword, además de dos videojuegos, Tzar: Excalibur y el rey Arturo y King Arthur: the role-playingwargame, con el objetivo de contribuir al estudio de la pervivencia del mito artúrico en la actualidad.

Palabras clave: rey Arturo, mitología, Historia, cine, videojuegos, adaptaciones.

\section{ABSTRACT}

The present work is proposed, first, to analyze the figure of King Arthur and the Knights of the Round Table, working on the main axes that make up the traditional mythological stories. In a second instance, the Arthurian imaginary will be addressed in films the Excalibur and King Arthur: Legend of the Sword, as well as two video games, Tzar: Excalibur and King Arthur and King Arthur: the role-playing wargame, with the aim of contributing to the study of the survival of the Arthurian myth today.

Keywords: King Arthur, mythology, History, cinema, video games, adaptations.

\section{INTRODUCCIÓN}

No cabe duda que la figura del rey Arturo ha trascendido todos los límites que la historia, la literatura, la mitología y la tradición poseen, sobreviviendo así al olvido de los tiempos. Ejemplo de ello es la contemporaneidad de nuestra época donde, luego de la narrativa - la pintura, el imaginario artúrico también se vio plasmado en el denominado séptimo arte, series televisivas y dibujos animados, para finalmente adentrarse en el mundo de los videojuegos. 
En este sentido, y en aras de contribuir conscientes al engrandecimiento de aquel mítico monarca, el objetivo de este trabajo será analizar el imaginario artúrico en las películas Excalibur y King Arthur: Legend of theSword, además de dos videojuegos, Tzar: Excalibur y el rey Arturo y King Arthur: the role-playingwargame.

\section{EL MITO ARTÚRICO}

Si bien la figura de Arturo está basada en una literatura de carácter mitológico, no faltan investigaciones que pretendan darle historicidad al señalarlo como un caudillo (bretwalda) del siglo VI que defendió el territorio contra los invasores sajones (Bryant, 2010; Brower, 2001; Fulton, 2009; Higham, 2002; Wheeler, 2004; Srigley Pirdum, 2010).

Dos obras tardías darán sustento a ese parecer histórico; por un lado, la Historia brittonum -atribuida a un tal Nennius- del siglo IX y los Anales Cambriae del siglo X. Sin embargo, resulta curioso que su nombre no se mencione en dos autores que son de vital importancia para el período de la Inglaterra anglosajona temprana; nos referimos a $\mathrm{De}$ exidiobritaniae de Gildas en el siglo v y la Historia ecclesiasticagentis Anglorum de Beda en el siglo VIII. Esta ausencia hizo y hace presuponer a los especialistas que, en realidad, Arturo no es más que una construcción mental y, luego, literaria.

Serán Geoffrey de Monmouth en el siglo XII y sir Thomas Malory en el siglo XV quienes construyan la leyenda o mitología de aquel valeroso, honrado y justo rey, como veremos a continuación.

Uther Pendragón, rey de Inglaterra, consiguió mediante la ayuda del mago Merlín adquirir la apariencia del duque de Cornualles, su vasallo, para seducir a la mujer de éste, lady Igraine, de quien estaba enamorado. El duque de Cornualles murió esa noche en batalla y Uther Pendragón e Igraine concibieron a Arturo. Meses más tarde, Uther se casó con Igraine, ahora viuda. A cambio de sus favores, Merlín solicitó a Uther la entrega de la criatura cuando naciera. Sin saber que era hijo del rey, Arturo fue educado por sir Ector, junto al hijo de este, sir Kay, quien fue como su hermano.

Poco después, el rey Uther fue atacado por pueblos enemigos y murió. El trono estuvo vacante durante mucho tiempo y el reino se tambaleó. Pasaron los años y un buen día se convocó a un torneo de caballeros en Londres, al que asistieron todos los del reino, incluido sir Ector con sus dos "hijos".

En el patio de la iglesia mayor apareció un gran bloque de mármol con la espada incrustada en él y una leyenda que decía: "Quien extraiga esta espada de la piedra es rey de Inglaterra por derecho de nacimiento".

Todos los caballeros se esforzaron por extraer la espada, pero no tuvieron éxito. En un momento del torneo, le tocó el turno a sir Kay, quien había olvidado su espada. Arturo se ofreció a buscarla. Por el camino tropezó con la espada en la piedra y la retiró sin esfuerzo.

En un principio Arturo no fue aceptado como rey, pues tenía 16 años y no lo consideraban hijo de Uther Pendragón. Finalmente, muchos reyes y duques aceptaron su derecho natural al trono, pero otros lo atacaron con sus ejércitos.

Para defenderse, Arturo contaba con la ayuda de Merlín, su fiel consejero, y de su espada mágica Excalibur. Poco después estableció su corte en Camelot y creó una orden de caballeros, nobles, aguerridos, dispuestos a defender toda causa justa y castigar a quienes ofendieran a un hombre indefenso o a una "inocente y gentil doncella". 
Una noche llegó hasta la corte una hermosa dama que dijo ser la esposa del rey Lot. La mujer sedujo al rey Arturo y juntos concibieron a un niño. Lo que Arturo no sabía es que esa mujer era Morgan Le Fay, su medio hermana (hija de su madre y de su primer esposo). Morgan Le Fay había aprendido todo acerca de magia, hechicería y nigromancia, y odiaba a Arturo por ser el heredero del trono. Arturo se enteró del nacimiento de su hijo producto del incesto y mandó colocar en una barca a todos los niños nacidos ese día. Sin embargo, la barca chocó contra unas rocas y un niño se salvó. Fue criado por unos campesinos, para convertirse años más tarde en sir Mordread, caballero de la Mesa Redonda y encargado de derrotar a su propio padre.

La unión de Arturo y Ginebra representa, a la vez, alegría y tristeza. Alegría porque ese amor fue correspondido mutuamente; pero tristeza porque ese matrimonio traería desgracia al reino, como se lo indicó en su momento Merlín al propio Arturo: "cásate con esa mujer y traerá desgracia a tu reino" (Kenney, 2010: 202-218; Radulescu, 2010: 326-339).

\section{MERLÍN, EXCALIBUR, LA MESA REDONDA Y EL SANTO GRIAL}

Al igual que muchas leyendas o mitos, Arturo y su contexto poseen elementos que son identitarios y que, independientemente del espacio en el que se represente, nunca faltan. Nos referimos a la figura de Merlín, la espada Excalibur, la Mesa Redonda y el Santo Grial. Por cuestiones de espacio, solamente daremos algunas consideraciones generales.

Cuenta la leyenda que Merlín (Bryant, 2010: 45-114; Le Goff, 2010: 143-150; Fulton, 2010: 84-101) era hijo de un duende perverso y de una princesa humana, concebido por medio de un ardid. Las hadas buenas lo habían tomado bajo su protección y, así, lo convirtieron en el más poderoso hechicero al servicio del Bien.

Mago (Fulton, 2010: 84-101) y sabio, viejo druida celta, Merlín tenía poderes que le diferenciaban de los demás mortales: conocía el futuro y las mentes de los hombres, podía adquirir la apariencia de un mendigo, de un muchacho o de un noble caballero. Sin embargo, no siempre podía modificar el destino, aunque lo conociera. Él construyó la Mesa Redonda y ubicó a cada caballero en su sitio. Fue consejero del rey Arturo y le enseñó a proteger a los pobres, los débiles y los indefensos.

La influencia de Merlín sobre los caballeros es, en general, de sabiduría y precaución. Pero los actos negligentes de los mortales pueden enojarlo $\mathrm{y}$, por este motivo, puede negarles su ayuda.

Al igual que Carlomagno y el Cid Campeador, Arturo también poseyó una espada de renombre, con la diferencia de que esta, Excalibur, fue forjada por el propio Merlín y, por lo tanto, poseía cualidades mágicas. Existen dos versiones sobre cómo el mítico rey la obtuvo. La primera cuenta que la tuvo luego de sacarla de una piedra en la que se hallaba clavada, y la otra es que un ser denominado la Dama del Lago se la cedió con la condición de que jamás la usara sin su vaina.

A pesar de cumplir con la condición de aquel ser, en la batalla contra Mordred, su cinturón se rompe y la vaina cae a la tierra. Arturo lucha y es herido de muerte, luego de haber vencido a su adversario. Al respecto, las leyendas narran dos historias. La primera es que, una vez finalizada la lucha, el rey es llevado junto al lago para, posteriormente, pedirle a uno de sus caballeros que arrojase la espada al agua, donde una mano blanca la tomó y la hundió para siempre. La otra historia cuenta que Excalibur descansa junto a Arturo en Avalon, hasta su regreso triunfal. 
La Mesa -construida por Merlín, como se ha mencionado-, de forma circular, tenía como objetivo evitar las disputas entre los caballeros que la componían. Los caballeros son: Gawaine, Tristán, Percival, Lancelot, Gareth, Galahard, Bedevere, Uwaine, Bors, Kay, Mordred, Tor (Putter, 2010: 235-251; Larrington, 2010: 252-264; Dalrymple, 2010: 265-277; Davenport, 2010: 278-294; Archibald, 2010: 312-325). No había jerarquías, salvo la del rey Arturo. La mesa era desmontable y el rey podía llevarla consigo donde fuera. Los caballeros recorrían los caminos de Europa cumpliendo su deber y de regreso a Inglaterra contaban aquellas aventuras.

Merlín escogió a los caballeros y ubicó a cada uno en su sitio. Solo una silla quedó vacía y en ella había un letrero que decía: "asiento peligroso". Sólo el caballero de alma pura, destinado a recuperar el Santo Grial, tendría derecho a ocupar la plaza vacante. En el castillo de Winchester se encuentra una réplica de la Mesa Redonda de Arturo. Es de roble, tiene seis metros de ancho y pesa una tonelada. En cada sitio tiene grabado el nombre de los caballeros correspondientes.

El último de los elementos clásicos artúricos es el Santo Grial. Según la tradición, el Santo Grial fue el cáliz en el que Jesús bebió durante la última cena. En él se guardó luego su sangre, derramada al ser herido en el costado cuando estaba crucificado.

La misma tradición nos señala que fue José de Arimatea (Bryant, 2010: 15-44) quien enterró el cuerpo de Jesús y se convirtió en el primer guardián del Santo Grial y de la lanza. Más tarde, José transportó estos bienes sagrados a Inglaterra, que quedaron al cuidado de sus descendientes, quienes fundaron la Orden del Grial.

En la época de Arturo el guardián de las reliquias era sir Pelles, rey del castillo de Carbonek. Entonces las reliquias desaparecieron; sólo aparecían en contadas ocasiones en las que sucedían grandes milagros a su alrededor. El Grial poseía muchas propiedades milagrosas, tales como proporcionar alimento a los que estaban libres de pecado o dejar libre al irreverente que llegaba a su presencia.

El Santo Grial sólo podría ser recuperado por un caballero de corazón puro. Todos los caballeros de la Mesa Redonda salieron en su búsqueda, pero el que estaba destinado a encontrarlo era sir Galahar. Él curó las heridas del rey Pelles y puso fin a las desgracias que asolaban la comarca. Luego se elevó al cielo junto al Cáliz, que así desapareció de la tierra para siempre.

\section{ARTURO Y EL CINE}

La Historia y el cine guardan, desde siempre, una estrecha relación que ha suscitado numerosas controversias en el ámbito académico, producto del rigor histórico que tienen -o no- las interpretaciones llevadas a la pantalla grande. En este sentido, el cine histórico es un relato de ficción, dado que lo concebimos como una manifestación literaria y artística, aunque también "algunos críticos y estudiosos consideran que, a pesar de esta ficción implícita, existen películas verdaderamente históricas" (Rodríguez, 2010:.31).

Si bien el cine constituye una representación de un período y/o personajes históricos, que oscila de manera permanente entre la veracidad y la ficción, consideramos que el pasado puede ser abordado desde ese punto si tenemos en cuenta que lo hará con sus propias particularidades. Así, las producciones cinematográficas nos permiten, por un lado, aproximarnos a un acontecimiento y/o personajes, y por el otro, nos brindan información 
sobre el contexto histórico en el que fueron realizadas (Rodríguez, 2010:30-31), tópicos que podremos ver presentes en las películas aquí tratadas.

Para ello, y dada la acotada extensión del artículo, hemos escogido dos films: en primer lugar Excalibur', una película de producción británica y estadounidense del año 1981, y en segundo lugar, King Arthur: Legend of the Sword ${ }^{2}$, que también cuenta con una coproducción británica-estadounidense, pero estrenada en este caso en el año 2017.

En este sentido, cabe mencionar que hemos optado por ambas dado que son producciones desarrolladas en contextos históricos y cinematográficos disímiles, tanto a nivel de la narrativa y de cómo el desarrollo de la tecnología contribuyó, a nuestro modo de ver, en el análisis que se realizó del mito del rey Arturo. Es decir, cómo la trama se fue transformando a lo largo del tiempo producto de las nuevas herramientas que el desarrollo tecnológico brinda a la industria del cine. A su vez, podemos ver cómo ambos films utilizan el mito artúrico y lo resignifican, seleccionando diversos elementos mitológicos para contar historias distintas.

Excalibur, de John Boorman, es un film basado en la obra de Sir Thomas Malory, La muerte de Arturo, publicada por primera vez en 1485, reuniendo las principales leyendas de origen francés e inglés sobre las historias de Arturo y sus Caballeros de la Mesa Redonda. El director del rodaje reconstruye la historia siendo fiel a los principales ejes que vertebran el mito artúrico, iniciando el film con las guerras desplegadas entre los barones ingleses en el período altomedieval con el fin de unificar el territorio y que el vencedor se coronase como rey.

Aquí podemos ver con claridad la configuración del poder político y de las relaciones de poder establecidas, características del período, en el que uno de los barones, Uther Pendragon, padre de Arturo, comienza a imponer su poderío sobre el resto. Es un poder fragmentado que comienza a ser acumulado en manos de uno de los barones, con claras relaciones de subordinación, en las que el rey debe ceder porciones de territorio a otros barones del reino a cambio de fidelidad, lealtad y el establecimiento definitivo de la paz. Este aspecto de la trama encierra un anacronismo histórico, dado que la correlación de poderes y la dinámica política aquí descriptas son en realidad características de siglos medievales posteriores al período en el que la película -y los relatos de Malory- se enmarcan.

En este contexto, el legendario mago Merlín ayuda a su amigo Uther concediéndole la espada de Excalibur-obtenida a través de la Dama del Lago-, símbolo de poder indiscutible dado que representa el origen del mundo conocido. Así, podemos ver cómo la espada constituye, en este punto, un elemento de conciliación, de paz, por el solo hecho de existir. Sin embargo, cuando Arturo logra sacarla de la roca donde su padre la había introducido, revelándose el origen del protagonista y convirtiéndolo en rey, el poder que Arturo posee sobre la espada no es razón suficiente para que ciertos barones le juren fidelidad al nuevo rey -como Lord Uryens, por ejemplo-. Para ello, Arturo deberá ganarse su confianza, construir su liderazgo demostrando su valentía y honor, mostrando ser digno de quien dice ser.

La historia del film transcurre presentando los principales hechos a los que el mito artúrico se refiere, desde la construcción de Arturo como rey, el establecimiento de la paz y

1 Página web para verla online: http://www.clasicofilm.com/2015/11/excalibur-1981-hdtv.html. Visitada por última vez el 02/03/2018.

2 Página web para verla online: http://miradetodo.io/king-arthur-legend-of-the-sword-aka-el-rey-arturola-leyenda-de-la-espada-2017-1080p-full-hd/. Visitada por última vez el 28/02/2018. 
la decisión de crear la tan famosa Mesa Redonda para sus caballeros, hasta su fallecimiento. Un aspecto característico de la película es que, en su devenir, la historia se va centrando en la vida de determinados personajes y en cómo la presencia de cada uno de ellos hace a la existencia misma del mito.

Por ello, el director dedica partes de la trama a ciertos personajes relevantes, tales como la aparición de Lancelot, su amistad con Arturo y su posterior historia de amor con Ginebra, esposa de Arturo. La relación entre Morgana, hermana de Arturo, y el mago Merlín también constituye uno de los nudos que conforman la trama, dado que en su devenir esta relación es utilizada por Morgana para hacer efectiva su venganza contra Arturo. En Excalibur, ella es quien encarna el papel de antagonista; es la bruja que utiliza sus encantos para engañar a Merlín con el objetivo de volverse una hechicera más sabia y poderosa, engañando a Arturo y logrando concebir un hijo suyo, Mordred. Iniciada una nueva etapa de hambrunas en el reino a causa de ello, Arturo envía a sus caballeros en busca del Santo Grial y, con él, del retorno de la prosperidad, búsqueda que se extiende por años hasta que Sir Parsifal halla el secreto perdido: Arturo, el rey, y la tierra "son uno", palabras pronunciadas también por Uther Pendragon cuando logró unificar el reino. En ambos, ese poder se hace factible al poseer y empuñar la espada de Excalibur.

Así, y con esta breve síntesis, podemos ver cómo Excalibur, en sus 140 minutos de duración, nos cuenta muchas historias en una sola, desarrollando también la de ciertos personajes que son relevantes en el mito del rey Arturo y que lo conforman como tal.

Por su parte, en King Arthur: Legend of the Sword, la perspectiva de la que parte el director Guy Ritchie es disímil; aquí podemos ver que la trama está centrada en una sola historia: la develación del origen real de Arturo y el proceso interno que él debe atravesar para aceptarlo, aceptarse a sí mismo y poder, así, luchar contra el usurpador de la corona: su tío Vortigern (Henshall, 2008: 92-96).

En esta producción, las transformaciones que la tecnología provocó en la industria cinematográfica en estas últimas décadas se hacen patentes, dado que los efectos especiales abundan y reemplazan, incluso, el desarrollo de otras tramas que bien podrían ser contadas dentro de esta historia. Así, Excalibur constituye un film mucho más rico en cuanto a las historias que narra y utiliza efectos especiales en ciertos momentos -de óptima calidad para el contexto de su filmación- que contribuyen al desarrollo de la narración, mientras que en King Arthur: Legend of the Sword los efectos especiales hacen a la película.

La historia, a su vez, no es fiel a los principales ejes del mito artúrico. En primer lugar, cabe mencionar que aquí Arturo nace en un matrimonio legítimo, el del rey Uther Pendragon y su esposa, a diferencia de Excalibur, donde es presentado como fruto de una infidelidad -producto, a su vez, de un engaño- entre el rey Uther e Igrayne, la esposa del Duque de Cornwall.

Otra característica particular es que el personaje antagónico es, como mencionamos con anterioridad, Vortigern, tío de Arturo, quien traicionó y asesinó a su hermano Uther para robarle la corona. Esta traición es llevada a cabo con ayuda de Mordred, que logra asesinar al rey de los magos a cambio de compartir el poder con su aliado. A este personaje también se le adjudica una identidad diferente a la brindada por el tradicional mito, donde se afirma que es hijo de Arturo y de Morgana, como pudimos ver en Excalibur, mientras que en King Arthur es un mago poderoso con una gran ambición de poder que libera a las fuerzas oscuras aliadas. 
Inicia, así, una etapa oscura en un reino ya unificado desde los inicios del rodaje, a diferencia del otro film, en el que la película comienza con las guerras que se dirigen hacia su fin, en busca del establecimiento de la paz. Los magos se ven sumidos en la oscuridad y deben permanecer en ella durante largos años para poder sobrevivir, hasta que se devela la verdad sobre el origen de Arturo. Un aspecto a remarcar es que el mago Merlín, un ícono en la mitología artúrica, no aparece en esta película. Su nombre es mencionado como el gran hacedor de la espada de Excalibur, pero su figura no se hace presente. En su lugar, hallamos un nuevo personaje: la Maga, una mujer que posee un vínculo especial con la naturaleza y que guía a Arturo en su camino, pero que no forma parte de las tradicionales leyendas.

Para finalizar, debemos mencionar que la relación entre Arturo y la espada de Excalibur es muy diferente en las dos películas. En la primera, podemos ver cómo Arturo acepta rápidamente su origen, su historia, su destino y a Excalibur, aprendiendo a invocar sus poderes para construirse a sí mismo como un rey valiente y honorable. Sin embargo, en el segundo film, Arturo se resiste sistemáticamente a admitir quién es y la conexión que posee con la espada, producto de su origen real y de su pertenencia al linaje de los Pendragon, desvaneciéndose cada vez que la empuña y teniendo visiones sobre la trágica muerte de su padre. Al aceptar su destino, Arturo puede vencer, finalmente, a su tío Vortigern y convertirse en el legendario rey Arturo.

\section{ARTURO Y LOS VIDEOJUEGOS}

La mitología artúrica, además de haber sido llevada a la pantalla grande, también tiene sus reinterpretaciones en el mundo de los videojuegos. Originados con el fin de ocupar un lugar en los momentos de ocio y distensión de una parte de la sociedad, los videojuegos han llegado para quedarse. A raíz de ello, en las últimas décadas, su desarrollo también ha encontrado una brecha en un universo cultural más amplio, que incluye una estrecha relación que ha ido estableciéndose entre los videojuegos y la Historia, en la medida en que los videojuegos aluden a ella a través de ciertas representaciones, poniendo en juego la verosimilitud dado que no siempre siguen un rigor histórico estricto (Coronado Schwindt y Gerardi, 2015: 104-105), como veremos en los casos que aquí abordaremos.

La novedad del videojuego radica en el hecho de que las historias contadas pueden ser asumidas en primera persona por los jugadores, "interactuamos con personajes y situaciones de época, llegando incluso a modificarla como divertimento: no olvidemos en ningún instante que se trata de un elemento de ocio. Y lo podemos ver y hacer a través de una pantalla de TV o de PC" (Jiménez Alcázar y Rodríguez, 2015: 303).

Es en este punto en el que podemos hallar una diferencia entre los videojuegos y el cine. ${ }^{3}$ Por ejemplo, dado que "el medio cinematográfico es finito, delimitado y concreto: su guion y definición del propio producto con límite temporal así lo evidencian. El videojuego no es así; las nuevas formas no lineales de los guiones hacen que se separe bastante del tradicional desarrollo de un film" (Jiménez Alcázar, 2016: 15).

En este apartado, pues, nos detendremos a analizar cómo el mito del rey Arturo y sus Caballeros de la Mesa Redonda ha sido reinterpretado y llevado al mundo de los

3 Una explicación más acabada de la relación puede leerse en Quaderns de cine 12. Cine y videojuegos, 2017, disponible en https://revistes.ua.es/quacine/issue/view/2017-n12-cine-y-videojuegos 
videojuegos a partir del abordaje concreto de Tzar: Excalibur y el rey Arturo ${ }^{4}$, del año 2002, y de King Arthur: the role-playingwargame ${ }^{5}$, del año 2009.

En el primer caso, el juego conforma una de las campañas de Tzar: theburden of the Crown, un videojuego de estrategia en tiempo real con numerosas ediciones, cada una de ellas ambientada en torno a una temática determinada. Tzar: Excalibur y el rey Arturo constituye la tercera campaña, conformada, a su vez, por ocho misiones en las que se van desarrollando ciertos ejes de las leyendas artúricas. Así, podemos ver que en cada una de ellas se recuperan los principales personajes y elementos que hacen al mito, como el legendario mago Merlín, los Caballeros de la Mesa Redonda, la espada de Excalibur, el Santo Grial, Morgana, Mordred, Camelot y los barones que se rebelan.

Un aspecto a resaltar es que si bien el marco histórico está dado por las historias del rey Arturo, el juego permite crear partidas combinando tres civilizaciones, tal como las presenta: la europea, la árabe y la asiática, a elección del jugador al momento de comenzar su partida, cada una con niveles de dificultad disímiles. La opción de partida libre permite, a su vez, que el jugador cree sus propias campañas, enfrentándose a ciertos enemigos en un mapa para tratar de vencerlos.

El objetivo final es conquistar nuevos territorios, expandiendo y fortaleciendo la civilización a través de la construcción de un imperio constituido por ciudades bien gobernadas, con una óptima administración de recursos económicos y militares que permitiera continuar con las expansiones. La cultura celta es también recuperada aquí, dado que los objetos mágicos y el arte de utilizar la magia son opciones a las que el jugador puede acceder, invocando criaturas míticas, tales como orcos y dragones que pueden ser utilizados para atacar al ejército enemigo o bien como defensa de las ciudades del reino.

King Arthur: the role-playingwargame, por su parte, pertenece al género que combina la estrategia en tiempo real con el juego de roles, tal como su nombre lo indica. La historia se centra en el reclamo de Arturo al trono de Britania, escenario geográfico en el que se desarrolla la trama, y que es presentado a través de un gran mapa topográfico en tres dimensiones, donde los héroes pueden acampar, conquistar provincias o buscar nuevas aventuras.

El jugador personifica al rey Arturo, reclutando caballeros para la Mesa Redonda con el fin de construir y defender su imperio. La campaña se divide en capítulos, cada uno de ellos con objetivos propios, que pueden ser a corto o largo plazo, y todas las decisiones a tomar por el o los jugadores -se puede jugar en modo individual o multijugador de manera directa en el desarrollo de la historia, es decir, en cómo se desenvuelve el mito artúrico en sí. Las misiones se visualizan regularmente en un mapa de campaña; constituyen aventuras que pueden llegar a convertirse en batallas a jugar en tiempo real, desplegadas en mapas especiales.

Así, si bien las historias mitológicas sobre el rey Arturo propician el marco histórico sobre el que el juego se desarrolla, el devenir del mito y el cauce que puedan tomar los sucesos quedan enteramente a disposición de las decisiones que el o los jugadores tomen en tiempo

4 Videojuego desarrollado por la empresa búlgara Haemimont Games. En España e Italia fue distribuida por FX Interactive. Esta campaña fue publicada en el año 2002.

5 Videojuego desarrollado por la empresa húngara NeocoreGames, lanzado en el año 2009. También posee una secuela titulada: King Arthur II: the role-playingwargame, publicada entre 2011 y 2012.

6 Para profundizar en el modo "multijugador" y sus características, véase Jiménez Alcázar, op. cit., pp. $30-32$. 
real; elecciones que habilitan -o no- ciertos contenidos especiales, tales como héroes, aliados y líneas de juego a seguir, así como también hechizos, dado que la magia también constituye uno de los aspectos que los productores del juego retoman del legendario mito.

El juego de roles es visible, a su vez, en los caballeros, quienes poseen habilidades que pueden ser mejoradas a través de puntos de experiencia y de cómo resuelven las misiones asignadas. Las elecciones y acciones que llevan a cabo en ellas afectarán a la Tabla de Moralidad, pudiendo convertirse, por ejemplo, en héroes o tiranos. Entre esas decisiones, pueden optar por cambiarse señores que gobiernan determinadas provincias o bien, pueden contraer matrimonio con damas que pertenecen a la corte del rey Arturo. Ambas acciones permiten que las relaciones de lealtad y fidelidad entre el rey y sus caballeros aumenten, aspecto que nos habla del contexto histórico en el que se enmarca el mito artúrico y del anacronismo al que aludimos con anterioridad.

En este breve apartado hemos visto cómo el mundo de los videojuegos ha ido apropiándose del legendario mito del rey Arturo y ha ido reinterpretándolo para ofrecer a los jugadores escenarios que presentan, como vimos en los casos aquí mencionados, grandes diferencias, que tienen que ver básicamente con la finalidad de cada videojuego, pero también ciertas similitudes que pueden resumirse en la recuperación y perpetuación de las principales historias y personajes de las leyendas artúricas, permitiendo que la esencia del mito artúrico aún perviva.

\section{CONCLUSIONES}

Algunas reflexiones parciales concluyen este trabajo indicando que la figura de un mito o una leyenda, una vez enraizado en el imaginario colectivo de cualquier sociedad o tradición, es muy difícil de escindir incluso a través del devenir temporal. Ejemplo de ello es el rey Arturo, un paradigma que ha transitado siglos y tradiciones estimando aquellos valores de justicia, sabiduría y carnadura humana.

A lo largo del presente trabajo hemos podido ver cómo tanto el mundo cinematográfico como el de los videojuegos ha ido tomando, a través del tiempo, las historias mitológicas artúricas, apropiándose de ellas para resignificarlas y dotarlas de un sentido propio, concreto y determinado, que guarda una estrecha relación con las características propias de cada género.

Por ello, en los casos aquí analizados, que nos han servido a modo de ejemplo, pudimos ver que los ejes y los aspectos que se recuperan de las tradicionales leyendas del rey Arturo pueden distar mucho entre sí: se recuperan personajes, objetos, historias específicas en cada uno de ellos, presentando ciertas similitudes, pero también algunas diferencias, todas ellas mencionadas con anterioridad. No obstante, tanto el cine como los videojuegos poseen un aspecto en común: su objetivo central es recuperar la mitología artúrica, permitiendo la pervivencia de la esencia del mito del rey Arturo y sus Caballeros de la Mesa Redonda hasta nuestros días.

Para finalizar, cabe mencionar que la pervivencia del mito artúrico no solo continúa readaptándose a las nuevas tecnologías, sino que prosigue manteniendo aquellos ideales caballerescos de una Edad Media remota y romántica. 


\section{BIBLIOGRAFÍA CITADA}

Archibald, E., "Malory's Lancelot and Guenevere", en Fulton, H. (Ed.), A Companion to Arthurian Literature, Blackwell, West Sussex, 2009, pp. 312-325.

Bryant, N. (Tras.), Merlin and the Grail. Joseph of Arimathea.Merlin.Perseval. The trilogy of prose romances attributed to Robert de Boron, D.S. BROWER, Cambridge, 2001.

Coronado Schwindt, G. y Gerardil, J. M., "Los videojuegos como productos culturales", en: Detchans, G. y Ferguson, J. (comp.): Humanidades y nuevas tecnologías, Colección Historia y Videojuegos n 4, Mar del Plata, 2015, pp. 104-105.

Dalrymple, R., "Sir Gawain in Middle English Romance", en Fulton, H. (Ed.), A Companion to Arthurian Literature, Blackwell, West Sussex, 2009, pp. 265-277.

Daventport, T., "The Medieval English Tristan", en Fulton, H. (Ed.),A Companion to Arthurian Literature, Blackwell, West Sussex, 2009, pp. 278-294.

Fulton, H., "Arthur and Merlin in Early Welsh Literature: Fantasy and Magic Naturalism", en Fulton, H. (Ed.), A Companion to Arthurian Literature, Blackwell, West Sussex, 2009, pp. 84-101.

Henshall, K., Folly and Fortune in Early British History.From Caesar to the Normans, Palgrave MacMillan, Gran Bretaña, 2008, pp. 92-96.

Higham, N., King Arthur. Myth-making and History, Routledge, London-New-York, 2002.

Jiménez Alcázar, J. F., De la Edad de los Imperios a la Guerra Total: Medioevo y Videojuegos, Colección Historia y Videojuegos n 3, Compobell, S.L. España, 2016.

Jiménez Alcázar, J. F. y Rodríguez, G., "¿Pasado abierto? El conocimiento del pasado histórico a través de los videojuegos". Pasado Abierto n² 2, Mar del plata, 2015.

Kenney, E. D., "The Graul and Franch Arthurian Romance", en Fulton, H. (Ed.), A Companion to Arthurian Literature, Blackwell, West Sussex, 2009, pp. 202-218.

Larrington, C, "English Chivalry and Sir Gawain and the Geeen Knight", en Fulton, H. (Ed.), A Companion to Arthurian Literature, Blackwell, West Sussex, 2009, pp. 252-264.

Le Goff, J., Héroes, maravillas y leyendas de la Edad Media, Madrid, Editorial Paidós, 2010, pp. 143-150.

Putter, A., "Arthurian Romance in English popular Tradition: Sir Percyvell of Gales, Sir Clerges, Sir Launfal", en Fulton, H. (ed.), A Companion to Arthurian Literature, Blackwell, West Sussex, 2009, pp. 235-251.

QUADERNS DE CINE 12. Cine y videojuegos, 2017, en https://revistes.ua.es/quacine/issue/ view/2017-n12-cine-y-videojuegos

Radulescu, R. L., "Malory and the Quest of the Holy Grail", en Fulton, H. Ed.), A Companion to Arthurian Literature, Blackwell, West Sussex, 2009, pp. 326-339.

Rodríguez, G., Ni Cenicienta ni princesa encantada: miradas actuales de la Edad Media, Editorial Cultura Fusión, Mar del Plata, 2010.

Srigley Pyrdum, C., Arthur the Bastard King: Legitimacy and Inheritance in the Arthurian Literature of Medieval England, University of Georgia, 2000, MA.

Wheeler, B. (Ed.), Arthurian Studies in honour of P. J. C. Field, S.D. BREWER, Cambridge, 2004. 


\section{FILMOGRAFÍA}

Excalibur,de John Boorman, 1981. Página web para ver online: http://www.clasicofilm. com/2015/11/excalibur-1981-hdtv.html. [Visitada por última vez el 02/03/2018.]

King Arthur: Legend of theSword, de Guy Ritchie, 2017. Página web para ver online: http:// miradetodo.io/king-arthur-legend-of-the-sword-aka-el-rey-arturo-la-leyenda-de-laespada-2017-1080p-full-hd/. [Visitada por última vez el 28/02/2018.]

\section{VIDEOJUEGOS}

King Arthur: the role-playingwargame, 2009. Videojuego desarrollado por la empresa húngara NeocoreGames. También posee una secuela titulada: King Arthur II: the roleplayingwargame, publicada entre 2011 y 2012.

Tzar: Excalibur y el rey Arturo, 2002. Videojuego desarrollado por la empresa búlgara HaemimontGames. En España e Italia fue distribuida por FX Interactive. 